\title{
Clinical Validation of a Virtual Environment Test for Safe Street Crossing in the Assessment of Acquired Brain Injury Patients with and without Neglect
}

\author{
Patricia Mesa-Gresa ${ }^{1}$, Jose A. Lozano ${ }^{1}$, Roberto Llórens ${ }^{1}$, Mariano Alcañiz ${ }^{1,2}$, \\ María Dolores Navarro ${ }^{3}$, and Enrique Noé ${ }^{3}$ \\ ${ }^{1}$ Universidad Politécnica de Valencia, Instituto Interuniversitario de Investigación en
} Bioingeniería y Tecnología Orientada al Ser Humano, LabHuman, Camino de Vera, s/n, 46022, Valencia, Spain

${ }^{2}$ Ciber, Fisiopatología Obesidad y Nutrición, CB06/03, Instituto de Salud Carlos III, Spain \{pmesa, jlozano, rllorens, malcaniz\} @labhuman. i3bh.es

${ }^{3}$ Servicio de NeuroRehabilitación. Hospital NISA Valencia al Mar y Sevilla Aljarafe, Fundación NISA, Valencia, Spain

loles@neurorhb.com; enoe@comv.es

\begin{abstract}
Acquired brain injury (ABI) is a complex disease that involves loss of brain functions related to cognitive and motor capabilities and that can produce unilateral spatial neglect (USN). The heterogeneity of the symptoms of these disorders causes a lack of consensus on suitable tools for evaluation and treatment. Recently, several studies have initiated the application of virtual reality (VR) systems as an evaluation instrument for neuropsychological disorders. Our main objective was to evaluate the validity of the VR Street Crossing Test (VRSCT) as an assessment tool. Twenty-five patients with ABI were evaluated with traditional tests and with the VRSCT. The results showed significant correlations between the conventional tests and the measures obtained with the VRSCT in non-negligent patients. Moreover, the VRSCT indicated significant differences in performance of negligent and non-negligent subjects. These pilot results indicate that ABI patients with and without USN can be assessed by the therapists using the VRSCT system as a complementary tool.
\end{abstract}

Keywords: Acquired brain injury, unilateral spatial neglect, pencil-and-paper tests, cognitive assessment, virtual reality, rehabilitation.

\section{Introduction}

The term 'Acquired Brain Injury' (ABI) refers to a normally developed brain that is damaged afterward. The causes can be diverse, including brain trauma, ischemic or hemorrhagic stroke, brain tumors, anoxia, etc. [1][2]. The main consequences of ABI involve the loss of brain functions that are related to the motor and cognitive systems as well as the loss of communication skills and emotional and behavioral regulation. There is also an important number of patients that currently have a long-term or lifelong need for help to perform activities of daily living (ADL) [1][2]. Brain injured 
patients may suffer cognitive difficulties such as problems with attention, memory, concentration, and executive functions (planning, judging, reasoning, etc.). Taking this heterogeneity of symptoms into account is essential to be able to carry out a proper assessment of the disease in order to ensure the success of the treatment.

Unilateral Spatial Neglect (USN) is a frequent disorder that is detected after brain damage [1]. The main feature of USN is the inability of patients to pay attention to stimuli located on the contralateral side of injury, and these symptoms are not related to sensorial or motor deficits [3][4]. This alteration may have important functional consequences for patients in their daily lives that make recovery more arduous. Patients who suffer symptoms usually fail to attend to their left/right side thereby disturbing their personal space (for example, shaving only half of their face), peripersonal space (reading one side of the two pages of a newspaper), and extrapersonal space (bumping into objects when walking) [5]. The complexity and heterogeneity of the symptoms of USN cause a lack of consensus on suitable tools for evaluation and treatment [4]. Although many cases of USN can be detected by observation, certain specific diagnoses require a specific evaluation to measure the severity of symptoms and possible progression. Traditional tools for assessment are based on pencil-and-paper tests as well as behavioral batteries [5][6]. The effectiveness of evaluation tests of this kind have been proved, although there are some limitations that must be taken into account such as the difficulty of interpreting the results, the extrapolation of results to daily life tasks, the difficulty of differentiating between sensory deficits, the lack of attention that is typical of USN, and the absence of assessment of changes in personal space [4][5].

In the last few decades, new procedures using virtual reality (VR) technologies have emerged. VR is a new technology based on computerized-generated stimulation that immerses the user in a realistic 3D world with multisensory stimuli and that offers the possibility to interact with the elements and receive feedback [4][7][8]. This technology, which has demonstrated its validity in the area of neuropsychology [2], can overcome some of the limitations of traditional evaluation methods. The main advantages of using VR are that it permits the evaluation and treatment in a realistic environment that is related to daily life and that is safer and has intrinsic ecological validity [5][7]. VR can generate different environments that allow more interaction and sense of presence and that improve the motivation of the users while enabling a precise control of each session to be maintained [8]. In the same way, the performance of the subjects can be recorded from different measuring procedures, which allows for individualized and adapted sessions in accordance with the limitations of the subject and/or the previous results obtained by the VR system [7][8]. In the case of the assessment of USN, the use of visual trackers by VR systems enables the eye movements of patients and their visual search pattern to be evaluated, which is important in learning more about the characteristics of this disease [5].

In recent years, several studies have initiated the application of VR systems as an evaluation tool for neuropsychological disorders. Some studies have carried out virtual versions of the classic pencil-and-paper tests. Fordell et al. [9] designed the VR-DiSTRO system which consists of a VR-test battery based on conventional tests for USN evaluation. The main results obtained in this study showed a high sensitivity of the VR system in detecting cases of neglect and a high level of agreement between measures of conventional tests and those obtained by the VR-DiSTRO. In another 
study [10], a semi-immersive work bench with stereoscopic glasses and a haptic device were used with a virtual version of the cancellation test. In this study, the results revealed that negligent and recovered patients showed irregular exploration performance in the VR task, with the VR system being more instructive than the conventional test. However, other VR systems include innovative diagnostic tasks. Dvorkin and colleagues [11] designed a Virtual Environment for Spatial Neglect Assessment application consisted of a 3D room-shape where patients had to respond when they detected a target (balls). The results indicated the sensitivity of the VR system for differentiating between negligent patients and control subjects, even though the similarity between the results of the VR system and a traditional test was less conclusive. More recently, Kim et al. [12] examined the efficiency of a 3D immersive virtual street crossing program for the assessment of post-stroke patients [7][13]. The VR system consisted in a real street crossing that could evaluate extrapersonal space in patients and showed significant differences between negligent and non-negligent patients.

A previous study by Navarro et al. [14] has demonstrated that our VR Street Crossing Test (VRSCT) is perceived by the patients as being a usable and satisfactory system in the rehabilitation of ABI and USN. Therefore, the main objective of our study is to evaluate the validity of the VRSCT system in terms of cognitive assessment. More specifically, the study compares the scores obtained from patients with ABI (with and without neglect) between different pencil-and-paper tests of attention and the measures found in the VRSCT system. Finally, we have tried to verify the sensitivity of our VR system as a complementary tool in neuropsychological assessment of attentional deficits in patients with ABI and with the diagnosis of USN.

\section{Materials and Methods}

Subjects. The participants of this study included twenty-five patients, 14 men and 11 women, aged $51.2 \pm 12.62$ years (mean \pm standard deviation or SD), with a mean chronicity of 505.42 days (SD: 335.11 ) and a mean of 11.84 years of education (SD:4.22). All the participants had sustained either a right or a left hemispheric brain lesion due to an ABI (hemorrhagic stroke: $n=12$, ischemic stroke: $n=10$, brain tumor: $\mathrm{n}=3$ ). The participants in this study were selected based on their scores on the MiniMental State Examination test (MMSE), which is a brief assessment of cognitive abilities, and an adapted version of the Mississipi Aphasia Screening Test (MAST), which evaluates comprehension of orders. All the subjects had a score greater than or equal to 24 on the MMSE and greater than or equal to 45 on the MAST. Therefore, participants had a cognitive level and proper comprehension to be able to handle the software.

VRSCT system. The VRSCT system simulates a typical city with its buildings, streets, cars, crosswalks, traffic lights, etc. In this environment, the objective of the patient is to go to a specific place in order to confront these adverse elements. This virtual environment is programmed to offer the therapist the possibility to configure it according to the difficulty level that is most appropriate for the patient. In order to 
offer the patient the virtual experience in an immersive way, we decided to use a conventional panoramic 47" LCD monitor, and a conventional 5.1 surround sound system. In order to interact with the virtual environment in an easy, intuitive and noninvasive way, we offered the patient two wireless devices: a conventional joystick for navigation and interaction, and an optical tracking system (TRACKIR, a product of NaturalPoint Company) to be able to track the patient's head movements. This last aspect is very important in the rehabilitation of neglect patients because they must get used to moving their heads to the neglect side of the space. The patient uses a cap that has three reflecting marks. The position and orientation of these marks are captured by a USB infrared camera that is placed in a panoramic LCD monitor. The device allows the patient to link his/her head movements in 3D space to the movement of the point of reference in the virtual environment. The device also allows these movements to be configured, for instance, linking small head-movements of the patient in 3D space to magnify the point of reference of movements in the virtual environment; this creates a panoramic view of the virtual environment of up to $180^{\circ}$.

In the experiment, the patients were placed in a quiet room where they sat at able in front of a widescreen monitor. A therapist trained in the virtual system was in the same room with the patient to give specific instructions about the assessment task and the software and to control the process. First, the patients received instructions on the use of the program. Then, the head tracking system was adjusted for each patient and a training session was administered to practice with the software and the hardware. For this training session, the patient had to navigate through the virtual streets and complete a single route without traffic or any other distractor. Once the patient had undergone the training session successfully, the assessment session began. The main goal of this session was to cross two-way roads in order to arrive at a destination point, a supermarket, and then return to the start point as quickly and as safely as possible. When an accident occurred, the patient received emotionally-intense audiovisual feedback (for example, sound of a car horn) and new instructions from the therapist. In this case, the program was automatically restarted from the initial point, without discounting the time already consumed. Taking into account previous studies, the session was considered completed when the patient performed two complete routes (each included arriving to the supermarket and getting back to the starting point) without more than four accidents. Therefore, when the patient did less than two complete routes or had more than four accidents, the patient was considered to have failed the task. The measures evaluated during the assessment session were the following: the number of times the participant looked to the left and to the right, the total time needed to finalized the task, the total number of accidents, and the completion/non-completion of the task (See Figure 1).
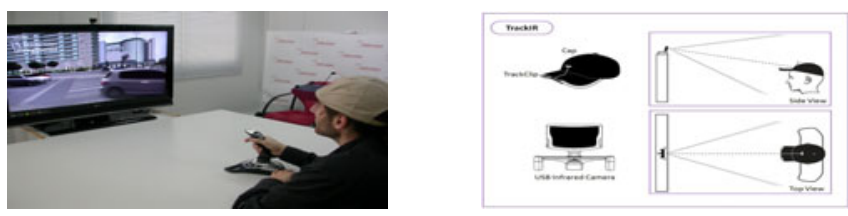

Fig. 1. Overview of the VRSCT and description of traking system 
Instruments. The pencil-and-paper tests used in the study are described below. The Behavioral Inattention Test (BIT) is a behavioral test battery that is designed to evaluate USN. It is composed of 15 subtests, 9 of which evaluate aspects of daily life and 6 of which are traditional tasks that assess neglect. The subtests of daily living are assessed by tasks like tracing line drawings, dialing, reading a menu, reading an article, telling and setting the time, choosing currencies, copying statements and addresses, map orientation and choosing cards. The evaluation of neglect on these tests consists of letter cancellation, star cancellation, a copying figures and shapes, line bisection and representative drawing [6]. To determine the presence of USN, the BIT scores were used taking the score of 129 as the cut-off. Based on the scores obtained by the participants and the normative scales of the test, the subjects were classified as "Negligent subjects" if the score was less than or equal to 129, and "Non-negligent subjects" if the score was greater than 129. The second test used was the Color Trail Making Test (CTT). This is a behavioral test that is based on the evaluation of sustained visual attention, mental flexibility, sequencing, visual tracking, and graphomotor ability. The CTT is a color version of the Trail Making Test. This test has two different parts: 1) Part A: the subject must connect the 25 numbers that are randomly distributed on a sheet with consecutive lines; and 2) Part B: There are 25 duplicate numbers within circles of different colors in which the subject must connect consecutive numbers with lines while at the same time alternating colors. The variable evaluated in this test is time (seconds) to perform each of the parts. Finally, the Conners' Continuous Performance Test-II (CPT-II) is a computerized test that evaluates the sustained attention of the subject and the ability to inhibit inappropriate responses. In this test, the subjects must press a key when they detect letters other than the letter ' $X$ '. The assessment contains 6 blocks that vary in the rate of submission of the letters. After testing, the program generates an automated report that includes data on various variables such as the number of omissions (letters that the subject did not mark), number of commissions (times that subject marked ' $\mathrm{X}$ '), reaction time (HIT Rt), and the capacity to adapt to the temporary demands of the task (HIT Rt ISI).

Procedure. All the patients were classified and selected based on their scores on the MMSE and the MAST. Once the 25 patients who participated in the study were selected, a cognitive evaluation was performed prior to using the VRSCT system. The cognitive assessment, which consisted of BIT, CTT, and CPT-II, was conducted during the same week as the virtual training. Then, evaluation and training with the VRSCT were initiated. The virtual test consisted of a preliminary training session and an evaluation session based on the task explained above. The training session took approximately 10 minutes and the evaluation session lasted until the patient finished the task and/or the patient was considered to have failed the task.

Data analyses. All statistical analyses were performed by SPSS 17.0 for Windows. The Pearson parametric correlation was used to analyse the BIT scores that classified patients as negligent or non-negligent in relation to the total time for the completion of the task in the assessment session of the virtual program and the number of accidents. The Pearson parametric correlation for continuous variables was used to measure the numerical scores obtained on tests of attention assessment (CTT and 
CPT-II) by non-negligent subjects that were related to the variables obtained by the VRSCT (total time to finalize the task and the number of accidents). The measures evaluated during the assessment session on the VRSCT were compared between negligent and non-negligent patients using the Mann-Whitney U-test. All the data are presented as mean \pm SD. In all cases, the significance levels were set at $\mathrm{p}<0.05$.

\section{Results}

Correlation between the BIT and the VRSCT system with negligent and non-negligent patients. According to the BIT scores, five of the twenty-five patients were classified as negligent subjects. The results obtained for non-negligent subjects $(n=20)$ showed a negative correlation that is statistically significant between the score obtained on the BIT and the total time needed to finalize the VRSCT $\left(r^{2}=-0.507, p<0.05\right)$ and the total number of accidents $\left(r^{2}=-0.515, p<0.05\right)$ (See Table 1 and Figure 2 a). None of the VRSCT measures were significantly correlated with the BIT score in negligent subjects.

Correlation between the conventional test for assessing attention (CTT and CPT-II) and the VRSCT system with non-negligent patients. On the CTT, scores obtained by non-negligent patients in part $\mathrm{A}\left(\mathrm{r}^{2}=0.802, \mathrm{p}<0.001\right)$ and part $\mathrm{B}\left(\mathrm{r}^{2}=0.506, \mathrm{p}<0.05\right)$ correlated significantly with the total time needed to finalize the VRSCT (See Figure 2 b). On the CPT-II test, only the significant correlation was between the HIT Rt ISI variable of the test and the total time needed to complete the task of the VRSCT $\left(\mathrm{r}^{2}=\right.$ 0.613, p<0.01). Other measures obtained with the CPT-II test (omissions, commissions, and HIT Rt) did not reach a significant correlation with the variables of the VRSCT.

Table 1. Correlation between the pencil-and-paper tests (BIT, CTT and CPT-II) and the variables of VRSCT for negligent and non-negligent subjects

\begin{tabular}{lrrrrc}
\hline & \multicolumn{2}{c}{ BIT } & CTT-A & CTT-B & $\begin{array}{c}\text { CPT HIT Rt } \\
\text { ISI }\end{array}$ \\
\cline { 2 - 6 } & $\begin{array}{c}\text { Negligent } \\
\text { vbjects }\end{array}$ & $\begin{array}{c}\text { Non-negligent } \\
\text { subjects }\end{array}$ & $\begin{array}{c}\text { Non-negligent } \\
\text { subjects }\end{array}$ & $\begin{array}{c}\text { Non-negligent } \\
\text { subjects }\end{array}$ & $\begin{array}{c}\text { Non-negligent } \\
\text { subjects }\end{array}$ \\
\hline $\begin{array}{l}\text { Total time (sec) } \\
\text { Number of }\end{array}$ & 0.704 & $-0.507^{*}$ & $0.802^{* *}$ & $0.506^{*}$ & $0.613^{* *}$ \\
accidents & -0.231 & $-0.515^{*}$ & 0.243 & 0.429 & 0.151 \\
\hline$* \mathrm{p}<0.05 * * * \mathrm{p}<0.01$ & & & &
\end{tabular}

Comparison of negligent and non-negligent patients' performances on the VRSCT. The total number of accidents registered in the VRSCT was significantly higher in negligent patients than in non-negligent patients $(U=17.000, p<0.05)$. Non-negligent patients showed a lower number of accidents $(3.00 \pm 1.41)$ than the negligent patients $(1.15 \pm 1.27)$. However, the total time needed to finalize the task in the VRSCT did not reach statistical significance between negligent and non-negligent patients $(p=0.734)$. 


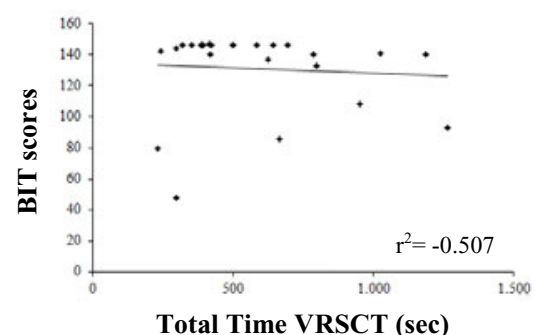

(a)

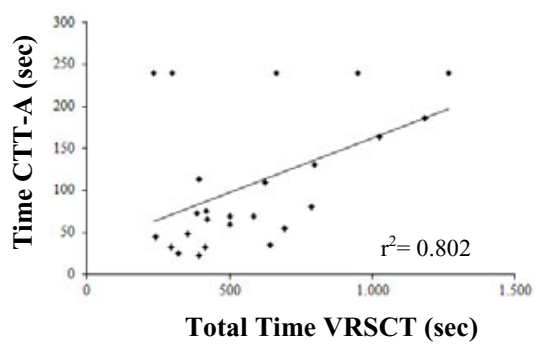

(b)

Fig. 2. (a) Correlation between the BIT scores and the total time needed to finalize the VRSCT in non-negligent patients. (b) Correlation between CTT-A scores and the total time needed to finalize the VRSCT in non-negligent patients.

\section{Discussion}

In this study, we evaluated the validity of VRSCT for the assessment of both negligent and non-negligent ABI patients. The results of the study showed that the VRSCT system was effective in terms of cognitive assessment. The measures obtained by the VRSCT system correlated with BIT score for non-negligent patients. More specifically, subjects that showed a lower score on the BIT spent more time completing the VRSCT task and had a higher number of accidents. However, with regard to the negligent patients, the results showed no significant correlation with the BIT data. Despite these result, the relationship between the scores of the BIT test and the VRSCT system in non-negligent patients may indicate that the virtual system measurements could be sensitive to the subject's attentional capacities. Future studies could make a more accurate comparison between the two types of diagnoses (i.e., traditional vs. VRSCT), by taking into account the subtasks of the BIT and expanding the measures assessed by the virtual system such as reaction time or time required for specific task performance.

The results also show that the VRSCT system has a positive correlation in nonnegligent patients with other traditional tests used to assess attention. More precisely, patients who took less time to solve the CTT and presented higher capacity to adapt to the temporary demands of the tasks of CPT-II had fewer accidents and spent less time completing the task in the VRSCT.

With respect to differences in the implementation of the VRSCT between negligent and non-negligent patients, the VRSCT showed significant differences in the measure of total number of accidents. The results show that the negligent patients had a higher number of accidents than non-negligent patients. Moreover, the system requirements to complete the task can evaluate the capacity of the patient to have an appropriate emotional response to the dangerous stimuli in daily life as well as the capacity of the patient to have an adequate reaction time. These pilot results indicate that the VRSCT can be used as a complementary tool for the diagnosis of patients with ABI affected by USN. We hope that this study is a first step in the construction of a complete platform for diagnosis and rehabilitation of ABI. Indeed, future studies and the implementation of improvements would allow the VRCST system to respond to the needs of both patients and therapists. 
Acknowledgments. This study was funded by Ministerio de Educación y Ciencia Spain, Project Game Teen (TIN2010-20187) projects Consolider-C (SEJ2006-14301/PSIC), "CIBER of Physiopathology of Obesity and Nutrition, an initiative of ISCIII" and Excellence Research Program PROMETEO (Generalitat Valenciana. Conselleria de Educación, 2008-157).

\section{References}

1. Ríos-Lago, M., Benito-León, J., Paul, N., Tirapu-Ustárroz, J.: Neurophychology of Acquired Brain Injury. In: Tirapu-Ustárroz, J., Ríos-Lago, M., Maestú, F. (eds.) Neuropsychology Handbook, Viguera, Barcelona, pp. 311-341 (2006)

2. Rose, F.D., Brooks, B.M., Rizzo, A.A.: Virtual Reality in Brain Damage Rehabilitation: Review. Cyberpsychol. Behav. 8, 241-261 (2005)

3. Dawson, A.M., Baxbaum, L.J., Rizzo, A.A.: The Virtual Reality Lateralized Attention Test: Sensitivity and Validity of a New Clinical Tool for Assessing Hemispatial Neglect, pp. 77-82. IEEE, Los Alamitos (2008)

4. Tsirlin, I., Dipierrix, E., Chokron, S., Coquillart, S., Ohlmann, T.: Uses of Virtual Reality for Diagnosis, Rehabilitation and Study of Unilateral Spatial Neglect: Review and Analysis. Cyberpsychol. Behav. 12, 175-181 (2009)

5. Ting, D.S.J., Pollock, A., Dutton, G.N., Doubal, F.N., Ting, D.S.W., Thomson, M., Dhillon, B.: Visual Neglect Following Stroke: Current Concepts and Future Focus. Surv. Ophthalmol. 56, 114-134 (2011)

6. Peña-Casanova, J., Gramunt Fombuena, N., Gich Fullà, J. (eds.): Neurophychological Tests. Elsevier, Barcelona (2006)

7. Katz, N., Ring, H., Naveh, Y., Kizony, R., Feintuch, U., Weiss, P.L.: Interactive Virtual Environment Training for Safe Street Crossing of Right Hemisphere Stroke Patients with Unilateral Spatial Neglect. Disabil. Rehabil. 29, 177-181 (2005)

8. Peñasco-Martín, B., De los Reyes-Guzmán, A., Gil-Agudo, A., Bernal-Sahún, A., PérezAguilar, B., De la Peña-González, I.: Application of Virtual Reality in the Motor Aspects of Neurorehabilitation. Rev. Neurol. 8, 481-488 (2010)

9. Fordell, H., Bodin, K., Bucht, G., Malm, J.: A Virtual Reality Test Battery for Assessment and Screenig of Spatial Neglect. Acta Neurol. Scand. 123, 167-174 (2011)

10. Broeren, J., Samuelsson, H., Stibrant-Sunnerhagen, K., Blomstrand, C., Rydmark, M.: Neglect Assessment as an Aplication of Virtual Reality. Acta Neurol. Scand. 116, 157163 (2007)

11. Dvorkin, A.Y., Rymer, W.Z., Harvey, R.L., Bogey, R.A., Patton, J.L.: Assessment and Monitoring of Recovery of Spatial Neglect within a Virtual Environment, pp. 88-92. IEEE, Los Alamitos (2008)

12. Kim, D.Y., Chang, W.H., Park, T.H., Lim, J.Y., Han, K., Kim, I.Y., Kim, S.I.: Assessment of Post-stroke Extrapersonal Neglect Using a Three-Dimensional Immersive Virtual Street Crossing Program. Acta Neurol. Scand. 121, 171-177 (2010)

13. Weiss, P.L., Naveh, Y., Katz, N.: Design and Testing of a Virtual Environment to Train Stroke Patients with Unilateral Spatial Neglect to Cross a Street Safely. Occup. Ther. Int. 10, 39-55 (2003)

14. Navarro, M.D., Alcañiz, M., Ferri, J., Lozano, J.A., Herrero, N., Chirivella, J.: Preliminary Validation of Ecotrain-Cognitive: A Virtual Environment Task for Safe Street Crossing in Acquired Brain Injury Patients with and without Unilateral Spatial Neglect. J. Cyber. Ther. Rehabil. 2, 199-203 (2009) 